\title{
Correlación Mastográfica E Histopatológica En Lesiones De Mama No Palpables
}

\author{
Bello-Sánchez Ma. Azucena \\ Especialista en Medicina Familiar, Unidad de Medicina Familiar No. 16 \\ Instituto Mexicano del Seguro Social, Delegación Querétaro, México \\ Villarreal-Ríos Enrique \\ Especialista en Medicina Familiar, \\ Unidad de Investigación Epidemiologia y Servicios de Salud \\ Instituto Mexicano del Seguro Social, Delegación Querétaro, México

\section{Blanco-Castillo Leticia}

Especialista en Medicina Familiar, Unidad de Medicina Familiar No. 16 Instituto Mexicano del Seguro Social, Delegación Querétaro, México

\section{Medina-Carreón Manuel}

Especialista en Ginecología y Obstetricia, Hospital General de Acapulco

Secretaria de Salud, Delegación Guerrero, México

\section{Reyes-Chávez Prishila Danae}

Especialista en Medicina Familiar, Unidad de Medicina Familiar 16. Instituto Mexicano del Seguro Social, Delegación Querétaro, México

\section{Martínez-Martínez Martha Leticia}

Especialista en Medicina Familiar.

Docente Investigador de la Facultad de Medicina,

Universidad Autónoma de Querétaro

\section{Camacho-Calderón Nicolás}

Especialista en Pediatría y Cardiopediatría.

Docente Investigador de la Facultad de Medicina,

Universidad Autónoma de Querétaro

\begin{abstract}
Globally, breast cancer ranks first in malignancies. It affects women over 40 years. An early diagnosis of no-palpable lesions, in sub-clinical stages, increases the probability of detecting at an early stage with increase survival and disease-free interval. Objective: To determine the correlation between mammography and histopatological lesions non palpable breast. Material and Methods: Study with correlational design. Histopathological records in women with non-palpable lesions and mamography of the
\end{abstract}


Mexican Social Security Institute, Querétaro, Mexico from 2011-2013. Sample size for correlational studies. Data obtained from the medical records of women with mammography BI-RADS 2 to 5 and confirmatory histopathology. Variables analized: sociodemographic data, family history of breast cáncer, histopathological diagnosis and mammography with BIRADS. Descriptive statistical analysis. $\mathrm{X}^{2}$ test and Cramer correlation with confidence level of 95\%. Results: 44 cases were analyzed. The average age of women was $52.04 \pm$ años (IC 95\%: 40.9 - 63.2). Predominant group of 56 to 70 years, $47.7 \%$ (IC 95\%: 36.5-58.9). Diagnosis with breast cancer 22.7\% (IC 95\%: 13.3 - 32.1), BI-RADS 2 in 4.5\% (IC 95\%: 0 - 9.1), BIRADS 3 in 29.5\% (IC 95\%: 19.3 - 39.7), BI-RADS 4 in 56.9\% (IC 95\%: 45.8 - 68.0) BI-RADS 5 in 9.1\% (IC 95\%: 2.7 - 15.5). The correlation between mammography and histopathological diagnosis was $0.26, p=0.43$. Conclusions: There was a low, no significant correlation, likely to include women with BI-RADS in early stage.

Keywords: BI-RADS, histopatological lesions, lesions non palpable breast, mammography

\section{Resumen}

A nivel mundial, el cáncer de mama ocupa el primer lugar en neoplasias malignas. Afecta a mujeres mayores de 40 años. Un diagnóstico oportuno de las lesiones no palpables, en fases subclínicas, aumenta la probabilidad de detectarlo en estadios tempranos con mayor supervivencia e intervalo libre de enfermedad. Objetivo: Determinar la correlación mastográfica e histopatológica de las lesiones de mama no palpables. Material y método: Estudio correlacional en registros histopatológicos de mujeres con lesiones no palpables y mastografía del Instituto Mexicano del Seguro Social, Querétaro, México del 2011 al 2013. Muestra estimada para estudios correlacionales. Se incluyeron expedientes de mujeres de la Clínica de Mama con resultados mastográficos BI-RADS 2 al 5 y con estudio histopatológico confirmatorio, análisis de variables sociodemográficas, antecedente de cáncer de mama familiar, reporte histopatológico y de BIRADS en la mastografía. Análisis estadístico descriptivo. Prueba de $\mathrm{X}^{2}$ y de correlación de Cramer con un nivel de confianza del 95\%. Resultados: Se analizaron 44 expedientes. El promedio de edad de las mujeres fue de 52.04 \pm 8.7 años (IC 95\%: 40.9 - 63.2). Predominó el grupo de 56 a 70 años, 47.7 \% (IC 95\%: 36.5-58.9). Diagnóstico de cáncer de mama el 22.7\% (IC 95\%: 13.3 - 32.1). BI-RADS 2 en el 4.5\% (IC 95\%: 0 - 9.1), BI-RADS 3, 29.5\% (IC 95\%: 19.3 - 39.7), BI-RADS 4, 56.9\% (IC 95\%: 45.8 - 68.0) BI-RADS 5, 9.1\% (IC 95\%: 2.7 - 15.5). La correlación entre la mastografía y diagnóstico histopatológico fue del 0.26 , con $p$ de 0.43 . Conclusiones: Hubo 
una correlación baja, no significativa, probablemente por incluir mujeres con BI-RADS en estadios tempranos.

Palabras clave: BI-RADS, lesiones histopatológicas, lesión mamaria no palpable, mastografía

\section{Introducción}

Las lesiones de la glándula mamaria corresponden a un grupo de alteraciones en el tejido mamario, las cuales pueden o no evolucionar a un proceso maligno, como respuesta a mecanismos de tipo hormonal, hábitos nutricionales y estilos de vida que interactúan entre sí, creando un grupo de signos y síntomas muy variados y manifestándose en la población principalmente femenina, como lesiones palpables y no palpables (Calderón, 2010).

Se define como lesión no palpable o subclínica, al nódulo que no es detectable mediante la autoexploración de la paciente ni clínicamente por el médico; aunque sí por estudios de tamizaje o por campañas de detección precoz del cáncer de mama a través de estudios de gabinete (Hernández, 2007).

La importancia de este tipo de lesiones es que pueden tener un comportamiento benigno cuando son nodulaciones tales como: mastopatía fibroquística, papilomas, hiperplasias y en el peor de los escenarios, lesiones malignas, como el carcinoma infiltrante o in situ, por lo que es necesario catalogarlas para indicar el tratamiento oportuno a cada tipo de lesión (Legido, 2010).

La prevalencia de cáncer de mama en las lesiones mamarias no palpables es de 50 a $60 \%$ en países con gran experiencia en programas de detección, como Holanda. En México, según lo reportado por el Hospital General de México, es de 35.6\% y en el Hospital Central Norte de Petróleos Mexicanos del 18.7\% (Ruvalcaba, 2009).

Las lesiones subclínicas de la mama corresponden a los hallazgos en estudios mamográficos o sonográficos que no se expresan clínicamente. La utilización cada vez más frecuente de los métodos de imagen para tamizaje para un diagnóstico y tratamiento temprano y oportuno de estas neoplasias requieren de mayor asiduidad ante la presencia de alteraciones mamográficas o ecográficas sospechosas (Lazcano, 2008).

La mastografía constituye en la actualidad la mejor prueba disponible para la detección y el diagnóstico precoz del cáncer de mama con una sensibilidad del 77 al 97\% y especificidad del 81 al 94\% (Torres-Arreola, 2007). La mastografía de escrutinio identifica lesiones no palpables, teniendo como alternativa una vez detectado el hallazgo anormal, realizar un análisis histopatológico, previa marcación de la lesión con arpón, biopsia con aguja 
gruesa y guía por ecografía o estereotaxia (Peralta, 2008; Vega, 2011; Duarte, 2012).

Estudios previos han reportado una correlación patológicamastográfica del 45\% (Vaquero-Pérez, 2002).

Con la finalidad de mejorar la efectividad y cobertura de las actividades preventivas del cáncer de mama, el presente trabajo determina la correlación mastográfica e histopatología de las lesiones de mama no palpables.

\section{Texto principal:}

Estudio con diseño correlacional a partir de registros histopatológicos de mujeres con lesiones no palpables y mastografía de la Clínica de Mama del Instituto Mexicano del Seguro Social, Querétaro, México, del 2011 al 2013.

El tamaño de la muestra $(n=44)$, se calculó con la fórmula para estudios correlacionales simple con un nivel de confianza del $95 \%$ y margen de error de $5 \%$.

Se incluyeron expedientes de mujeres con lesiones de mama no palpables y con resultados de mastografía e histopatología interpretados y reportados por médico radiólogo y patólogo respectivamente. Fueron excluidos los expedientes con diagnóstico previo de cáncer de mama. Se eliminaron expedientes clínicos con datos incompletos.

Se analizaron las variables: sociodemográficas (edad, ocupación y estado civil), antecedentes de cáncer de mama en la madre, hermana o abuela, reporte histopatológico y el resultado de la mastografía con BIRADS del 2 al 5.

Se analizó la información con estadística descriptiva (promedios y desviación estándar), frecuencias absolutas y relativas e intervalos de confianza al $95 \%$, inferencial con la prueba de $\mathrm{X}^{2}$ y para la asociación entre variables nominales se correlacionó con la prueba de Cramer.

\section{Resultados}

Se revisaron 44 expedientes clínicos de mujeres con lesiones no palpables de glándula mamaria. El promedio de edad fue de 52.04 años (IC 95\%: 40.9 -63.2), predominó el grupo de 56 a 70 años en $47.7 \%$ (IC 95\%: 36.5-58.9), dedicadas al hogar 68.2\% (IC95\%: 57.8-78.6) y casadas $90.9 \%$ (IC95\%: 84.5-97.3).

Con antecedente de carga familiar para neoplasias ginecológicas 13.6\% (IC95\%: 5.9-21.3), con mayor frecuencia en la abuela.

De las pacientes con lesión no palpable, se diagnosticaron con cáncer de mama el 22.7\% (IC 95\%: 13.3-32.1); de éstas, el 100\% correspondió a carcinoma ductal infiltrante (Véase Cuadro 1). 
El BI-RADS 4 fue la clasificación que predominó en el 56.9\% ( Véase Cuadro 2)

La relación entre la mastografía y la posibilidad de presentar o no cáncer de mama fue significativa (Véase Cuadro 3).

Los estudios mastográficos e histopatológicos presentaron una correlación de Cramer de 0.26, con una $p>0.05$ (Véase Cuadro 4).

\section{Discusión}

En la consulta de primer nivel de atención médica se dispone de dos métodos para el tamizaje de la patología mamaria: la exploración clínica y la mastografía.

Hay escenarios en que la paciente no tiene expresión clínica de las lesiones y el reporte mastográfico reporta una imagen sugestiva de malignidad, por lo que es necesario un método definitivo que esclarezca el diagnóstico, como lo es la biopsia. Bajo este contexto clínico, se ubica la importancia de abordar el tema de la correlación entre la mastografía e histología en las lesiones no palpables de mama.

En relación al grupo etario, las mujeres cursaban la cuarta década de la vida que coincide con lo reportado por Rubalcaba (2009) y Vara (2011), en que las tenían BI-RADS de 3 y 4 . Esto implica que se tiene que detectar en forma temprana el cáncer de mama en este grupo de la población femenina tal como lo enfatiza Peralta (2008) como el grupo de mayor riesgo para el desarrollo y fallecimiento por cáncer de mama.

De acuerdo al antecedente familiar, existe mayor probabilidad de que se exprese esta variante de patología mamaria en las mujeres que tengan familiares de primera línea y que hayan presentado algún tipo de cáncer. En esta investigación se tuvo el antecedente por parte de la abuela, situación que alerta en relación con los antecedentes familiares maternos para el desarrollo de esta patología (Calderón, 2010).

La mastografía como método fundamental requiere de la calidad de la imagen y la experiencia del médico radiólogo que la interpreta ya que influye en la categorización de las calcificaciones. En este estudio, la mayoría de las pacientes fueron incluidas dentro del BI-RADS 4, es decir, con imágenes sugestivas de neoplasia. Sin embargo, Silva (2012), considera que siempre existe una excepción a la regla, por lo que, en algunos casos las calcificaciones con altas sospecha de malignidad resultan con reporte histopatológico benigno.

De acuerdo a los lineamientos de la NOM-041-SSA-2011 para la prevención, diagnóstico, tratamiento, control y vigilancia epidemiológica del cáncer de mama, el BI-RADS 2 está considerado como una etapa clínica benigna. En esta serie, dos pacientes con esta categoría se les diagnosticó cáncer de mama. Este hecho implica que debe de realizarse los 
lineamientos en esta norma oficial además de indagar los factores de riesgo biológico o estilo de vida no saludable que puede modificar negativamente el curso de estas lesiones mamarias.

La mastografía aún cuando tenga una alta sensibilidad y especificidad, es un método de tamizaje, no de diagnóstico (Manual operativo para el uso de la mastografía). Peralta (2008), Vega (2011) y Duarte (2012) citan que una vez que se ha detectado una imagen anormal, se requiere de análisis histopatológico, por lo que será indispensable la toma de biopsia en estas lesiones no palpables.

Generalmente los reportes histológicos muestran que las lesiones que no tienen expresión clínica, usualmente cursan con patología mamaria de tipo benigno, siendo en la mayoría de los casos la mastopatía fibroquística, aunque existe un porcentaje menor a $25 \%$, que pueden ser precursoras de malignidad (Tejerina, 2012); dicha aseveración se expone en esta investigación , al revelar que alrededor del $20 \%$ de la población, fue positiva para el cáncer de mama. Bajo este contexto, no se deben de subestimar las condiciones $\mathrm{y}$ ofrecerles a las usuarias estudios de gabinetes complementarios para establecer un diagnóstico y tratamiento oportuno que limite el desarrollo del proceso oncológico.

Se encontró una correlación baja no significativa estadísticamente. Hubo factores que se han considerado para este resultado; uno de ellos es el tamaño de muestra para conformar un subgrupo de acuerdo al BI-RADS, el considerar un BI-RADS 2 y no BI-RADS 3 en la que se considera como sospecha de malignidad (Hernández, 2007).

El presente estudio aborda un tema relevante y de importancia epidemiológica en las mujeres por los factores de riesgo ya identificados para el desarrollo de cáncer de mama. Se requieren de estudios de mayor población que permitan establecer una correlación significativa de acuerdo a la clasificación de BI-RADS y los hallazgos clínicos sugestivos, en este caso las lesiones no palpables que representan potencialmente casos de cáncer.

\section{Conclusion:}

Se encontró una correlación baja y no significativa entre la mastografía e histopatología de las lesiones de mama no palpables en esta población de mujeres de la Clínica de Mama.

\section{References:}

Calderón VG, Pimentel CK, Camones YR, Cotrina CJ, Vigil RC, Velarde GR, et al. "Diagnóstico y tratamiento de las lesiones no palpables de la mama en el Instituto Nacional de Enfermedades Neoplásicas durante el año 2010”. Acta cancero Núm. 40, Vol. 1, pp 23-30, Lima, Perú. 2012. 
Duarte C, Bastidas F, Reyes A, Manrique J. "Localización radioguiada de las lesiones ocultas de la mama”. Revista Colombiana de Cancerología Núm. 16, Vol. 4, pp 239-243. Colombia. 2012.

Hernández NI, Sandoval GF, Hernández GM, Torres LA, Martí RJ, Ríos RN. "Lesiones de mama no palpables sospechosas de malignidad. Correlación radiológica-quirúrgica (Hospital General de México, OD)". Gamo Núm. 6, Vol. 2, pp 42-46. México, D.F. 2007.

Lazcano MA, Peralta A, Reyes Y, Redondo F, Ramos M, Guerrero G. "Correlación histopatológica de las Microcalcificaciones diagnosticadas por mastografía en el Hospital General de México de enero del 2007 a diciembre del 2008". Anales de Radiología México Núm. 3, pp. 42-46. México, D.F. 2009.

Norma Oficial Mexicana NOM-041-SSA-2011. "Para la prevención, diagnóstico, tratamiento, control y vigilancia epidemiológica del cáncer de mama”. México. 2011.

Peralta A, Lazcano A, Reyes Y, Redondo F, Marti J, Ríos N, et al. "Reporte de lesiones no palpables diagnosticadas por mastografía en el Hospital General de México". Anales de Radiología México Vol. 4, pp.248251. México, D.F. 2008.

Ruvalcaba LE, Espejo FR, Bautista PV, Madero PL, Capurso GM, José Eduardo Serratos GJE. 2009. "Control radiológico intraoperatorio de una pieza quirúrgica en lesiones mamarias no palpables”. Ginecol Obstet Mex Núm. 77, Vol. 9, pp 407-418. México. 2009.

Secretaria de Salud. Guía de Práctica Clínica: Diagnóstico y tratamiento del Cáncer de mama en segundo y tercer nivel de atención. México, D.F. 2009.

Torres-Arreola LP, Vladislavovna SV. "Cáncer de mama. Detección oportuna en el primer nivel de atención". Rev Med Inst Mex Seguro Soc Núm. 45, Vol. 2, pp 157-166. México, D.F. 2007.

Vaquero-Pérez MA, Pérez-Robledo JP, Corripio R, Granado de la Fuente JM, Moreno-Azcoita M. "Supervivencia del cáncer de mama diagnosticado como lesión mamaria no palpable”. Cir Esp Núm. 72, Vol. 1, pp 10-13. La Paz, Madrid. 2002.

Vara-Salazar E, Suárez-López L, Ángeles-Lllerenas A, Torres-Mejía G, Lazcano-Ponce E. "Tendencia de la mortalidad por cáncer de mama en México, 1980 -2009”. Salud Pública Méx Núm. 53, Vol. 5, pp. 385-393. México D.F. 2011.

Silva L. "Hallazgos histopatológicos de calcificaciones BI-RADS 4 en el Hospital General de México: reporte de casos". Anales de Radiología México Núm. 3, pp. 150-156. México D.F. 2012.

Vega BA. "Intervencionismo diagnóstico en patología de mama". Radiología Núm. 6, Vol. 53, pp. 531-543, Santander, España 2011. 
Yépez-Ramírez D, De la Rosa AG, Guerrero-Albarrán C, Gómez-Martínez JM. "Autoexploración mamaria: conocimiento y perspectiva en mujeres". Rev Enferm Inst Mex Seguro Soc Núm. 20 Vol. 2, pp 79-84. México D.F. 2012.

Patricia Legido Moran. Lesiones de mama no palpables. Disponible en: www.departamentocirugiavalladolid.com/LibroHomenajedeAmistad.pdf, pp 157 -178. Consultado el 5 de diciembre del 2013.

Cuadro 1. Frecuencia de acuerdo al hallazgo de estudio histopatológico.

\begin{tabular}{c|cccc} 
Entidad patológica. & Frecuencia & Porcentaje & \multicolumn{2}{c}{ IC 95\%* } \\
\cline { 4 - 5 } & & & Inferior & Superior \\
\hline Mastopatía fibroquística & 27 & 61.4 & 50.5 & 72.3 \\
Adenosis esclerosante & 1 & 2.3 & 0 & 5.6 \\
Fibroadenoma mamario & 6 & 13.6 & 5.9 & 21.3 \\
Carcinoma ductal & 10 & 22.7 & 13.3 & 32.1
\end{tabular}

Fuente: Sistema de registro de Patología, del HGR1, IMSS, delegación Querétaro, periodo enero 2011 - diciembre 2013.

\begin{tabular}{|c|c|c|c|c|}
\hline \multirow[t]{2}{*}{ BI-RADS } & \multirow[t]{2}{*}{ Frecuencia } & \multirow[t]{2}{*}{ Porcentaje } & \multicolumn{2}{|c|}{ IC $95 \% *$} \\
\hline & & & Inferior & Superior \\
\hline 2 & 2 & 4.5 & 0 & 9.1 \\
\hline 3 & 13 & 29.5 & 19.3 & 39.7 \\
\hline 4 & 25 & 56.9 & 45.8 & 68 \\
\hline 5 & 4 & 9.1 & 2.7 & 15.5 \\
\hline
\end{tabular}

Fuente: Sistema de registro del Servicio de Patología del HGR1, IMSS, Delegación Querétaro, enero 2011 - diciembre 2013.

Cuadro 3. Relación entre el resultado de la mastografía en paciente con y sin cáncer de mama.

\begin{tabular}{c|cccc}
\multirow{2}{*}{$\begin{array}{c}\text { Resultado de } \\
\text { mastografía }\end{array}$} & \multicolumn{2}{|c}{ Sin cáncer } & \multicolumn{2}{c}{ Con cáncer } \\
\hline 2 & Frecuencia & Porcentaje & Frecuencia & Porcentaje \\
3 & 1 & 50.0 & 1 & 50 \\
4 & 11 & 84.5 & 2 & 15.5 \\
5 & 21 & 84.0 & 4 & 16.0 \\
& 1 & 25.0 & 3 & 75.0
\end{tabular}

Prueba de $\mathrm{x} 2$ *p: 0.04

Fuente: Sistema de registro de Patología, del HGR1, IMSS, delegación Querétaro, enero 2011 - diciembre 2013. 
Cuadro 4. Relación entre el BI-RADS en mastografía y entidad histopatológica.

\begin{tabular}{|c|c|c|c|c|c|c|c|c|c|c|c|c|}
\hline \multirow{3}{*}{$\begin{array}{l}\text { Diagnóstico } \\
\text { histopatológic } \\
\text { o }\end{array}$} & \multirow{2}{*}{\multicolumn{3}{|c|}{2}} & \multirow{2}{*}{\multicolumn{6}{|c|}{ BI-RADS }} & \\
\hline & & & & & & & & & & & & \\
\hline & $\mathrm{n}$ & $\%$ & $\begin{array}{c}\text { IC95 } \\
\%\end{array}$ & $\mathrm{n}$ & $\%$ & $\begin{array}{c}\mathrm{IC} 95 \\
\%\end{array}$ & $\mathrm{~N}$ & $\%$ & $\begin{array}{c}\text { IC95 } \\
\%\end{array}$ & $\mathrm{n}$ & $\%$ & $\begin{array}{c}\text { IC95 } \\
\%\end{array}$ \\
\hline $\begin{array}{l}\text { Mastopatía } \\
\text { fibroquística }\end{array}$ & 1 & $\begin{array}{l}5 \\
0\end{array}$ & $\begin{array}{c}38.8- \\
61.2\end{array}$ & 9 & $\begin{array}{c}69 . \\
2\end{array}$ & $\begin{array}{l}58.9- \\
79.5\end{array}$ & $\begin{array}{l}1 \\
6\end{array}$ & $\begin{array}{l}6 \\
4\end{array}$ & $\begin{array}{l}53.3- \\
74.7\end{array}$ & 1 & $\begin{array}{l}2 \\
5\end{array}$ & $\begin{array}{l}15.3- \\
34.7\end{array}$ \\
\hline $\begin{array}{l}\text { Adenosis } \\
\text { esclerosante }\end{array}$ & 0 & 0 & 0 & 0 & 0 & 0 & 1 & 4 & $0-8.4$ & 0 & 0 & 0 \\
\hline $\begin{array}{c}\text { Fibroadenoma } \\
\text { mamario }\end{array}$ & 0 & 0 & 0 & 2 & $\begin{array}{c}15 . \\
4\end{array}$ & $\begin{array}{l}7.3- \\
23.5\end{array}$ & 4 & $\begin{array}{l}1 \\
6\end{array}$ & $\begin{array}{l}7.8- \\
24.2\end{array}$ & 0 & 0 & 0 \\
\hline $\begin{array}{l}\text { Carcinoma } \\
\text { ductal }\end{array}$ & 1 & $\begin{array}{l}5 \\
0\end{array}$ & $\begin{array}{c}38.8- \\
61.2\end{array}$ & 2 & $\begin{array}{c}15 . \\
4\end{array}$ & $\begin{array}{l}7.3- \\
23.5\end{array}$ & 4 & $\begin{array}{l}1 \\
6\end{array}$ & $\begin{array}{l}7.8- \\
24.2\end{array}$ & 3 & $\begin{array}{l}7 \\
5\end{array}$ & $\begin{array}{l}65.3- \\
84.7\end{array}$ \\
\hline & & & & $\begin{array}{r}\text { Pru } \\
\text { Prue }\end{array}$ & ba d & $\begin{array}{l}* \mathrm{p}: 0 \\
2: 9.04 \\
\text { amer: }\end{array}$ & & & & & & \\
\hline
\end{tabular}

Fuente: Sistema de registro de Patología, del HGR1, IMSS, delegación Querétaro, periodo enero 2011 - diciembre 2013. 